\title{
Influência do sistema silvipastoril na puberdade de novilhas
}

\author{
Graciele Araújo de Oliveira Caetano ${ }^{1 *}$ \& Messias Batista Caetano Júnior²
}

${ }^{1}$ Zootecnista, Mestre em produção animal - Universidade Federal dos Vales do Jequitinhonha e Mucuri ${ }^{2} Z$ ootecnista - Instituto Federal de Minas Gerais, Campus Bambuí

*Autor para correspondência, E-mail: caetanozootecnia@outlook.com

\begin{abstract}
RESUMO. Como consequência da preocupação atual com o esgotamento dos recursos naturais, aliado a um maior busca por melhores índices reprodutivos, a bovinocultura brasileira vem tomando medidas priorizando a precocidade sexual do seu rebanho. No Brasil, um país tropical, que possui um sistema de produção pecuário basicamente a pasto, o fator estresse calórico vem se tornando um fator limitante na sustentabilidade da pecuária brasileira. Elevadas temperaturas podem influenciar negativamente na principal característica reprodutiva indicadora da precocidade sexual de bovinos que é a idade a puberdade. Esse efeito negativo do calor (estresse) implica em ajustes fisiológicos no animal na busca da manutenção da homeotermia, entre eles ocorrem alterações no fluxo sanguíneo, comportamentais, na ingestão de alimentos e hormonais influenciando o início da puberdade. Rebanhos detentores de elevada precocidade aumentam a produção, antecipa a recuperação dos investimentos, aumenta a vida produtiva dos animais permitindo uma maior lucratividade. Na busca da sustentabilidade e competitividade da pecuária leiteira, as pesquisas vem apontando os sistemas silvipastoris que associam o cultivo de plantas lenhosas perenes, forrageiras e animais como uma boa opção. Isso é explicado pela formação de um microclima confortável termicamente, amenizando os efeitos danosos do estresse calórico na reprodução, além de favorecer o solo e a pastagem.
\end{abstract}

Palavras chave: Agroflorestal, estresse calórico, maturidade sexual, reprodução

\section{The silvopastoral's system influence on heifers' puberty}

\begin{abstract}
As a result of current concern with the depletion of natural resources, combined with a larger quest for better reproductive rates, the Brazilian cattle industry has been taking measures giving priority to sexual precocity of his flock. In Brazil, a tropical country, which has a production system basically livestock grazing, heat stress factor is becoming a limiting factor in the sustainability of cattle breeding. High temperatures can negatively influence the main reproductive characteristic indicator of sexual precocity in cattle is age puberty. This negative effect of heat (stress) implies physiological adjustments in the animal in search of maintaining homeothermy, among them are alterations in blood flow, behavior, food intake and hormonal factors influencing the onset of puberty. Livestock keepers, high precocity increase production anticipates the recovery of investments, increase the productive life of animals allowing for greater profitability. In search of sustainability and competitiveness of dairy farming, the research has been pointing the silvopastoral systems involving the cultivation of woody perennials, grasses and animals as a good option. This is explained by the formation of a thermally comfortable microclimate, mitigating the harmful effects of heat stress on reproduction, besides improving the soil and pasture.
\end{abstract}

Keywords: Agroforestry, breeding, heat stress, sexual maturity

\section{Introdução}

O desenvolvimento das atividades agropecuárias no Brasil sempre esteve associado à derrubada da cobertura florestal. Isso é evidenciado, desde o período colonial com os seus ciclos econômicos baseados na derrubada da mata nativa para a eventual exploração sem se 
preocupar com as consequências na reserva dos recursos naturais (Paciullo et al., 2007).

Nos dias atuais, a fiscalização e a preocupação com o esgotamento dos recursos naturais vêm dificultando a continuação dessa prática extrativista de produção. Isso vem obrigando o pecuarista a buscar alternativas para aumentar a produtividade integrando ações conservacionistas. Com isso, os sistemas multifuncionais (silvipastoris) têm sido apontados como uma forma adequada para solucionar esses problemas.

O sistema silvipastoril é uma das variações dos sistemas agroflorestais. Possui essa classificação por apresentar uma associação de árvore-pasto-animal num mesmo ecossistema. Essa interação entre os componentes traz vários benefícios tanto para conservação do ambiente como um incremento na produtividade $\mathrm{e}$ fertilidade.

Chama-se à atenção para a presença do componente arbóreo no sistema. Em decorrência do seu sombreamento forma-se um microclima ameno termicamente que favorece a criação animal (Meirelles, 2005). Essa zona térmica produzida é um dos principais benefícios observados no que diz respeito à pecuária.

O estresse calórico é um fator limitante na sustentabilidade da atividade reprodutiva dos bovinos, principalmente em países como o Brasil em que os animais são criados de forma extensiva e em temperaturas elevadas. Esse efeito negativo do calor (estresse) implica em ajustes fisiológicos no animal, na busca da manutenção da homeotermia acarretando em perdas na precocidade sexual. Entre esses ajustes ocorrem alterações no fluxo sanguíneo, comportamentais, na ingestão de alimentos e hormonais influenciando nas características reprodutivas como a idade à puberdade.

O sistema silvipastoril busca amenizar, através da formação de um microclima favorável, os efeitos deletérios do estresse calórico tanto na produção quanto na reprodução animal viabilizando a pecuária nos países tropicais.

\section{Sistema silvipastoril}

Os sistemas silvipastoris (SSP) são uma das diversas variações dos Sistemas Agroflorestais (SAF's). Segundo Carvalho (1998) são arranjos de técnicas alternativas de uso de solo, combinando espécies florestais, culturas agrícolas, atividades pecuárias, ou ambas, simultaneamente. Constitui uma forma alternativa de uso da terra e exploração agrícola, pela maior sustentabilidade biológica, econômica, social e ecológica, comparada com sistemas tradicionais, como o monocultivo de pastagens (Montagnini, 1992).

São também conhecidos como sistemas agroflorestais pecuários e se caracterizam por integrar componentes lenhosos (árvores e arbustos), herbáceos (gramíneas e leguminosas) e animais herbívoros (Carvalho et al., 2003).

Nos últimos anos, tem aumentado o reconhecimento dos benefícios potenciais gerados pelos sistemas silvipastoris, de modo que vários tipos de sistemas estão em estudo em diversas instituições de pesquisa do País. Começam a ser adotados em propriedades particulares e empresas comerciais, salientando a viabilização e sustentabilidade desse policultivo. É considerado como uma opção viável para aumentar a biodiversidade animal e vegetal, além de aumentar os níveis de produção animal com reduzida dependência de insumos externos (Paciullo et al., 2007).

\section{Microclima favorável ao Sistema silvipastoril}

Dentre as variáveis climáticas que afetam o comportamento animal, destaca-se a temperatura do ar, umidade do ar, velocidade do vento e radiação solar.

A presença de árvores nas pastagens, além de diminuir a incidência de radiação solar, reduz a temperatura do ar por meio da evapotranspiração que ocorre em suas folhas, também possibilitando mais adequada circulação do ar sob sua copa. Como consequiência, quando a sensação térmica a céu aberto estiver entre 36 e $40^{\circ} \mathrm{C}$, sob sombra natural será reduzida para 26 a $32^{\circ} \mathrm{C}$ (Pires et al, 2005).

O sombreamento é fator de grande importância para a produtividade bovina, principalmente na pecuária leiteira. Pesquisas têm demonstrado que ambientes de conforto e bem-estar podem refletir em melhor desempenho produtivo e reprodutivo dos animais (Pires et al, 2002). A provisão de sombra é uma das primeiras medidas tomadas para modificar o ambiente e proteger o animal dos efeitos da radiação solar, prevenindo $\mathrm{o}$ estresse calórico. Estudos concluíram que bovinos desconfortados, durante o verão, procuraram por ambientes sombreados diminuindo o tempo que seria gasto com pastejo 
e ruminação, indicando a necessidade de provisão de sombra (Pires et al., 2007).

A melhor sombra é aquela fornecida pelas árvores, pois há estudos que afirmam que a evaporação da umidade proveniente das folhas esfria até $8^{\circ} \mathrm{C}$ o ar sob a copa sem interferir na circulação do ar. Além disso, o calor radiante proveniente das folhas é muito pouco absorvido pelos animais quando comparado com coberturas de metal (Ferreira, 2005).

\section{Estresse calórico}

O estresse calórico (EC) pode ser definido como a somatória de forças externas ao animal homeotérmico que atuam de forma a alterar a temperatura corporal do estado de repouso (Yousef, 1984).

Os bovinos, animais homeotérmicos, devem manter a temperatura corporal dentro de limites estreitos ao longo das 24 horas do dia independente das temperaturas externas. Em determinadas situações ambientais, o animal pode manter todas as suas funções vitais (mantença, reprodução e produção) e, em outras, estabelece prioridades. À medida que o ambiente vai se tornando mais severo suas funções vão sendo suprimidas (Kadzere et al., 2002).

Todo animal possui uma zona de temperatura ótima para seu pleno desenvolvimento e produção. Essa zona é denominada Zona de Conforto Térmico (ZCT), definda como o intervalo existente na faixa de temperatura do ar circundante, onde a temperatura do corpo do animal mantém-se constante com um mínimo de esforço do seu centro termorregulador. $\mathrm{O}$ animal começa a sofrer estresse, quando estiver fora desses limites. A variação dessa temperatura seria entre 10 e $27{ }^{\circ} \mathrm{C}$ para o gado zebuíno (Bos indicus) adulto e entre 1 e $21{ }^{\circ} \mathrm{C}$ para o gado europeu (Bos taurus) (Gürtler, 1987).

Quando o animal é submetido às temperaturas fora dessa zona, ele passa a tomar medidas para tentar se adaptar as novas condições que está submetido. Dentre elas pode-se citar: um aumento na produção de suor, na frequiência cardíaca e respiratória, diminuição na ingestão de alimentos e na atividade corporal, busca por ambientes mais amenos (Gürtler, 1987).

\section{Estresse na reprodução}

Um animal submetido a uma situação estressante, como temperaturas fora da sua zona termoneutra, responde através da ativação do eixo hipotalâmico-hipofisário-adrenal (HHA). Essa ativação gera uma cascata de mensagens hormonais que culminam num aumento nas concentrações plasmáticas de CRH (hormônio liberador de corticotropina) e consequentemente de corticosteróides e catecolaminas (Rivier \& Rivest, 1991).

O CRH desempenha um papel importante na modulação dos efeitos do estresse sobre a função reprodutiva. Ele atua no hipotálamo através da inibição da secreção de GnRH; na hipófise, por interferência do GnRH na liberação de LH e FSH e por fim nas gônadas alterando sua responsividade às gonadotropinas (Rivier \& Rivest, 1991).

O hormônio liberador de gonadotropinas GnRH é produzido pelo hipotálamo, órgão localizado na base do cérebro, e age regulando a liberação das gonadotrofinas, Hormônio Folículo Estimulante - FSH e Hormônio Luteinizante LH. O FSH e o LH, produzidos pela glândula adenohipófise (hipófise anterior) são responsáveis pelo desenvolvimento folicular e ovulação (Hafez, 2004).

Como vimos alhures, para que aconteça a puberdade é necessário que ocorra a primeira ovulação acompanhada pelo desenvolvimento de um corpo lúteo capaz de se manter durante um ciclo estral completo. Em estresse prolongado observa-se a inibição das secreções de LH e FSH impedindo o crescimento e a maturação folicular bloqueando a ovulação.

Por outro lado, o estresse também acarreta mudanças comportamentais no animal. Em condições de altas temperaturas percebe-se uma redução no tempo que seria destinado ao pastejo (consumo de matéria seca), enquanto uma maior ingestão de água resulta numa distenção gástrica diminuindo o apetite. Esses fatores vem a contribuir para uma maior demora no ganho de peso dos animais no que se refere ao início da puberdade.

Concomitantemente, uma redução na ingestão de alimentos acarreta num decréscimo da secreção do sinalizador do status metabólico corporal, a leptina. Torna-se claro que a subnutrição aumenta a idade à puberdade diminuindo, consideravelmente, a eficiência reprodutiva do rebanho. 


\section{Precocidade sexual}

A precocidade sexual está intimamente ligada à eficiência reprodutiva e a lucratividade da pecuária bovina. Na atividade bovina, qualquer variação negativa nas características reprodutivas podem gerar atrasos econômicos cerca de dez vezes maior do que em características associadas ao crescimento. Rebanhos com altas taxas de fertilidade possuem maiores disponibilidades de animais, tanto para a venda como para intensificação seletiva do progresso genético acarretando em um maior lucro (Hill, 1998).

Um dos maiores desafios da pecuária nacional é tornar o zebu mais precoce, uma vez que as novilhas pertencentes ao rebanho brasileiro possuem elevada idade à puberdade variando entre 24 a 30 meses, bem acima do preconizado que seria entre 12 a 14 meses (ANUALPEC, 2005).

A principal característica indicadora da precocidade sexual de bovinos é a idade a puberdade. Tal importância pode ser vista nos programas de melhoramento dos zebus que utilizam este indicador como referencial nos ganhos genéticos. Rebanhos detentores de elevada precocidade aumentam a produção, antecipa a recuperação dos investimentos, aumenta a vida produtiva e permite um rápido ganho genético (Bergmann et al., 1996).

\section{Puberdade $x$ maturidade sexual}

O termo puberdade vem do latim pubescere que significa apresentar pêlos em função do aparecimento de pêlos púbicos em decorrência do aumento de andrógenos pela adrenal nesse período (Terasawa \& Fernandes, 2001).

Em mamíferos a puberdade é definida como o momento fisiológico em que o animal torna-se capaz de se reproduzir (início funcional dos órgãos reprodutivos), mesmo não tendo o eixohipotalâmico-hipofisário-gonadal (HHG) ainda totalmente regulado.

É reflexo da idade fisiológica (tamanho ou peso) e não da idade cronológica das fêmeas. As novilhas atingem a puberdade no primeiro cio, que é seguido de uma fase luteínica normal. Entretanto, a primeira ovulação não significa que o animal esteja sexualmente maduro (Jainudeen \& Hafez, 2004). Isso vem a corroborar que puberdade e maturidade sexual são coisas distintas. Essa diferença é atribuída a fatores genéticos, nutricionais e ambientais.
Um conceito importante associado à puberdade é a ocorrência do estro não puberal. Este estro recebeu esse nome devido ao fato de o animal apresentar comportamento de cio, sem, no entanto, ser seguido de ovulação, ou seja, a secreção de estradiol foi suficiente para a manifestação de estro, porém as concentrações foram inadequadas para induzir a ocorrência da ovulação. Este achado tem sido reportado em em torno de 13 a $22 \%$ dos animais, num período médio de 89 dias antes do estro puberal. Em alguns animais, pode ocorrer mais que um estro não puberal (Kinder et al., 1987; 1994; 1995).

Geralmente, são necessários 3 a 4 ciclos estrais com fases luteais normais, ou seja, 60 a 90 dias após a ocorrência da puberdade, para que a fêmea bovina atinja sua maturidade sexual e adquira plena capacidade de conceber e de levar a gestação a termo (Kinder et al., 1994). A idade média na puberdade de novilhas submetidas a níveis nutricionais recomendados é de 10 a 12 meses em raças leiteiras e de 11 a 15 meses em raças de corte. Já as novilhas zebuínas alcançam a puberdade entre 18 e 24 meses de idade (Schillo et al., 1992).

\section{Endrocrinolgia da puberdade}

A entrada na puberdade, sob o ponto de vista hormonal, tem sido definida como a primeira ovulação acompanhada pelo desenvolvimento de um corpo lúteo capaz de se manter durante um ciclo estral completo (Moran et al., 1989). Esta fase requer muito gasto de energia e nutrientes, uma vez que necessite garantir a manutenção e o crescimento de um novo indivíduo em uma eventual gestação.

O início das secreções das gonadotrofinas FSH (hormônio folículo estimulante) e LH (hormônio luteinizante) e de seus hormônios hipotalâmicos liberadores como o $\mathrm{GnRH}$ (hormônio liberador de gonadotrofinas) acontece, precocemente, $\log o$ ao final da diferenciação sexual (1 a 2 meses de gestação) (Day et al., 1984; 1987; Kinder et al., 1987; Mpran et al., 1989; Evans et al., 1994; Anderson et al., 1996).

Após o nascimento da fêmea, vários mecanismos evitam a ativação do sistema endócrino reprodutivo (primeira ovulação) antes de um desenvolvimento somático compatível para a reprodução. Embora esses eventos tenham sido bem documentados, pouco se conhece sobre os mecanismos que controlam o início da manifestação dos mesmos (Kinder et al., 1987). 
Diante disso, a hipótese gonadostática foi proposta para compreender esses eventos fisiológicos de contenção da atividade gonadal. Essa hipótese preconiza a existência de uma excessiva sensibilidade do hipotálamo ao estradiol $\left(E_{2}\right)$ com consequente exarcebação da retroalimentação negativa (Rodrigues et al., 2002).

Bezerras de 1 a 3 meses já possuem o HHG (eixohipotalâmico - hipofisário - gonadal) funcionalmente maduro, sendo capaz de promover eventos endócrinos que induzem ao estro. Porém, a alta sensibilidade do hipotálamo ao estradiol leva à inibição da atividade reprodutiva (Day et al., 1987). Nessa fase os ovários já estão ativos e já possuem folículos em crescimento o que geram uma produção de estrógenos incapaz de atingir o alto limiar para estimular a liberação do hormônio liberador das gonadotropinas $(\mathrm{GnRH})$ pelo hipotálamo (McDonald, 1989). Baixas concentrações de estradiol $\left(\mathrm{E}_{2}\right)$ exercem um feed-back negativo na liberação tônica de LH (Schillo et al., 1992).

Sem uma explicação plausível, a partir da décima semana de vida, estendendo-se até a vigésima segunda semana (2 a 5 meses), observase aumento gradativo na secreção de LH, seguido por um decréscimo (Nakada et al., 2001).

Semanas que antecedem a puberdade, ocorre um aumento na frequência de secreção do LH, resultado de uma diminuição do efeito inibitório do estradiol $\left(\mathrm{E}_{2}\right)$ sobre a secreção de $\mathrm{GnRH}$ pelo hipotálamo e uma diminuição de seu feed-back negativo diretamente na hipófise devido a mudança na quantidade de receptores de estradiol em diferentes áreas do hipotálamo. Isso acarreta num aumento da responsividade da neurohipófise ao GnRH (Rodrigues et al., 2002). Segundo Day et al. (1984) essa queda na retroalimentação negativa do estradiol sobre a liberação do LH diminui a medida que a puberdade se aproxima. Enquanto a secreção de FSH permanece inalterada (Nogueiraet al., 2003).

$\mathrm{O}$ aumento da frequiência dos picos de secreção de LH resulta em crescimento dos folículos e aumento da produção de estradiol folicular o que ira ocasionar o primeiro pico préovulatório e consequentemente a ovulação. Considerado como um pré-requisito para o início da puberdade (Nakada et al., 2000). Na grande maioria dos casos, apesar dessa ovulação ser considerada normal, não é uma certeza que a fêmea está madura sexualmente, além de não apresentar sinais externos de cio (Schillo, 1992).

Outras pesquisas vêm mostrando que outro fator que age na contenção gonadal e na conseqüente inibição da manifestação da puberdade seria as concentrações de $\mathrm{P}_{4}$. Quando a puberdade se aproxima aumenta a frequência dos pulsos de LH acarretando um ligeiro aumento nos níveis de progesterona (McDonald, 1989).

Segundo Gonzalez-Padilla et al. (1975), detectaram dois picos súbitos de progesterona que precedem a ocorrência da puberdade. Ainda está em questionamento a origem da progesterona detectada nestes dois picos. Durante a fase luteal curta, ocorre uma liberação de $\mathrm{P}_{4} \mathrm{em}$ concentração suficiente para sincronizar $\mathrm{o}$ desenvolvimento dos folículos e promover a sensibilização dos ovários à ação do LH garantindo que ocorra o segundo pico préovulatório do $\mathrm{LH}$, esse que resultará na ovulação (Evans et al., 1994).

Marson et al. (2001) ressalta que com o efeito inibitório do $\mathrm{E}_{2}$ seria necessário um estímulo anterior como a $\mathrm{P}_{4}$ para que se manifeste sinais externos do estro da puberdade seguido de uma ovulação e função luteal competente. Também é atribuído ao conjunto progesterona/estradiol mudanças morfológicas uterinas promovendo um ambiente mais propício ao desenvolvimento embrionário.

Entre os mecanismos responsáveis pelo controle da secreção e da pulsatilidade de LH, podemos destacar os neurotransmissores capazes de estimular, como os aminoácidos excitatórios (glutamato, aspartato), neuropeptídeo Y, noraepinefrina, noradrenalina e ácido aspártico; e neurotransmissores inibitórios, como o GABA, dopamina, opióides endógenos e endorfinas.

\section{Peso afetando a puberdade}

Como a nutrição tem efeito decisivo sobre a precocidade à puberdade, a obtenção do peso crítico é medida prioritária para o sucesso reprodutivo do sistema de produção de bovinos (Lobato, 1997).

O início da puberdade está mais intimamente relacionado ao peso corporal do que a idade. Em bovinos de corte sua puberdade é atingida quando seu peso representa de 45 a $50 \%$ do seu peso adulto (Schillo et al., 1992). Entretanto, há trabalhos que afirmam que o peso ideal à puberdade varia entre 60 e $65 \%$ do peso adulto 
(Spire, 1997). Segundo Silva et al. (1991) as fêmeas da raça Nelore apresentam médias ao redor de $300 \mathrm{~kg}$ para o peso ao primeiro cio (puberdade). De qualquer modo, o peso ideal ao início da puberdade varia muito de acordo com a raça tendo como principal fator de variação o plano nutricional da fêmea.

Vale ressaltar que um bom aporte nutricional influencia a idade à puberdade, mas não se consegue afetar o peso à puberdade.

A habilidade de uma fêmea em manter uma maior frequiência dos pulsos de liberação do $\mathrm{LH}$, necessários para o crescimento dos folículos e conseqüente a ovulação, está relacionada ao seu estado metabólico, ou seja, à sua reserva energética. Flutuações no metabolismo devido a mudanças tanto na massa qu na gordura corporal podem modular a liberação dos picos de LH. Observaram que os folículos de novilhas com dieta mais rica em energia atingiram tamanho suficiente à ovulação 63 dias antes que outras em dietas de baixo teor energético (Schillo, 1992).

Além disso, afirma-se que uma maior ingestão de alimentos pode ocasionar num aumento no volume hepático, no fluxo sanguíneo e na concentração de enzimas hepáticas que oxidam hormônios esteróides causando uma maior taxa de metabolização de hormônios esteróides. Isso resulta em uma redução do feed-back negativo à secreção de LH e FSH (Santos \& Santos, 2003).

A deficiência nutricional em novilhas de corte suprime, no hipotálamo, a geração de picos de secreção de LH atrasando a primeira ovulação (Schillo et al., 1983).

O sistema nervoso central (SNC) reconhece que o corpo atingiu o peso ideal para a reprodução. Essa sinalização assim como as variações associadas à primeira ovulação podem ser explicadas pela concentração de leptina circulante no organismo (GarciaA et al., 2002).

\section{Leptina e a puberdade}

A leptina é um produto do gene da obesidade, é uma proteína secretada pelos adipócitos e está implicada na regulação da ingestão de alimentos, no balanço energético e no eixo neuroendócrino dos bovinos e outras espécies animais. É considerada como um sinalizador do status metabólico corporal atuante no eixo hipotalâmico-hipofisário para o início da puberdade. Uma redução na ingestão de alimentos acarreta num decréscimo de sua secreção, numa queda da condição corporal coincidindo com uma diminuição na liberação de LH (Garcia et al., 2002).

Um possível mecanismo pelo qual a leptina age no eixo reprodutivo seria sua ligação ao neurotransmissor endógeno $\beta$-endorfina que por sua vez influencia positivamente os neurônios produtores de GnRH e negativamente os neuropeptídeos Y (NPY) envolvidos no controle da saciedade. Uma restrição alimentar prolongada resultará numa redução no escore da condição corporal, isso leva a uma queda na concentração de leptina circulante promovendo a elevação dos valores do neuropeptídeo Y e, desta forma, diminuindo a secreção de GnRH hipotalâmico. Há estudos que apontam a ação da leptina nas células- $\beta$ do pâncreas, estimulando a produção de insulina (figura 1) (Willians et al., 2002).

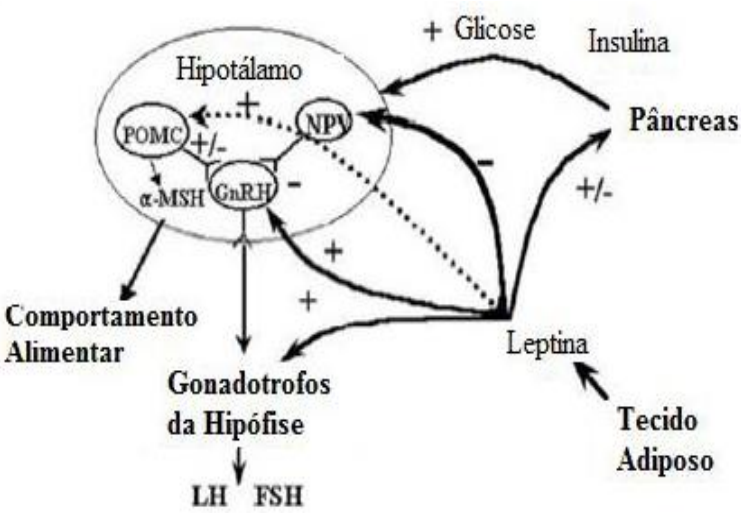

Figura 1. Efeitos da leptina; Fonte. (Willians et al., 2002)

Em animais subnutridos, os neuropeptídeos Y apresentam em grande quantidade no núcleo arcuado do hipotálamo. Essa região também contém neurônios cuja função é a secreção de GnRH. Esses neuropeptídeos interferem tanto na secreção de LH quanto no comportamento sexual devido à alta inervação dessa região (Willians et al., 2002).

A outra explicação para a ação da leptina como sendo um sinalizador metabólico do tempo da puberdade é a sua atuação no recrutamento de transportadores de glicose insulino-dependentes aumentando os níveis plasmáticos de glicose circulante. Desse modo, essa glicose disponível além de exercer suas funções na reprodução também atuaria em nível de sistema nervoso central, atuando nos glicosensores do hipotálamo induzindo a liberação de GnRH (Foster \& Nagatani, 1999). 
À medida que a puberdade se aproxima, observou-se um aumento nos níveis séricos de leptina, sendo tal fato seguido do aumento concomitante dos níveis de LH circulante (Garcia et al., 2002).

Porém, ainda existem controvérsias quanto à ação direta da leptina no aumento das frequiências dos pulsos de LH. Segundo Zieba et al. (2005) e Maciel et al. (2004) fica claro o papel permissivo da leptina no início da puberdade, entretanto é incapaz de aumentar diretamente os picos de LH em novilhas pré-púberes.

\section{Considerações finais}

A adoção dos sistemas de multicutivo tem sido usada como uma grande ferramenta no incremento da produtividade proporcionando múltiplos produtos aliado com os ideais conservacionistas bastante destacados, no cenário mundial atual.

O estresse térmico em bovinos é uma das principais causas da queda dos índices reprodutivos na atividade pecuária. Destaca-se a grande interferência na precocidade sexual do rebanho bovino, referindo-se principalmente na idade à puberdade. $\mathrm{O}$ Brasil por ser um país tropical e possuir mais de $85 \%$ do seu rebanho criado a pasto, esse elemento tem seus prejuízos intensificados ainda mais.

O sistema silvipastoril vem sendo cogitado como uma das melhores soluções atuais, no que se refere ao aumento da produtividade e melhora nos índices zootécnicos, principalmente, em rebanhos de países tropicais como o Brasil.

A adoção dessa prática ainda apresenta níveis muito baixos. Os estudos e as pesquisas ainda são incipientes comprovando a interferência do sistema silvipastoril na reprodução bovina, porém, o maior aprofundamento e entendimento no assunto irão proporcionar uma melhor percepção dos reais benefícios do modelo.

\section{Referências Bibliográficas}

Cardoso, D. \& Nogueira, G.P. Mecanismos neuroendócrinos envolvidos na puberdade de novilhas. Arquivos de Ciências Veterinárias e Zoologia da Unipar, v.10, n.1, p.59-67, 2007.

Carvalho, M. M.; Alvim, M. J.; Xavier, D. F.; Yamaguchi, L. C. T. Um sistema silvipastoril para recuperação de áreas degradas da Mata Atlântica. Juiz de Fora: Embrapa gado de
Leite, 2003b. 6 p. (Embrapa Gado de Leite. Comuincado Técnico, 31.).

Carvalho, M.M. Arborização de pastagens cultivadas. Juiz de Fora: EMBRAPA-CNPGL, 1998. 37p. (EMBRAPA CNPGL. Documentos, 64).

Ferreira, R.A. Maior Produção com Melhor Ambiente para Aves, Suínos e Bovinos. Ed. Aprenda Fácil, 2005, Viçosa - MG. p. 213239.

Foster, D.L. \& Nagatani, S. Physiological perspectives on leptin as a regulator of reproduction: role in timing puberty. Biology Reproduction, v.60, p.205-215, 1999.

Gonzalez-Padilla E, Wiltbank JN \& Niswender Gd. Puberty in beef heifers. Journal Animal Science, v.40, p.1091-1104, 1975.

Gürtler, H., Ketz, H.A., Kolb, E., Schröder, L. \& Seidel, H. Fisiologia Veterinária. Rio de Janeiro: Guanabara Koogan. 1987.

Hafez, E.S.E. \& Hafez, B. Reprodução Animal. 7.ed. Barueri: Manole, 2004. 513p.

Kadzere, C.T.; Murphy, M.R.; Silanikove, N.; Maltz, E. Heat stress in lactating dairy cows: a review. Livestock Production Science, v.77, p.59-91, 2002.

Kinder Je, Bergfeld, E.G.M. Wehrman, M.E., Peters, K.E., Kojima, F.N. Endocrine basis for puberty in heifers and ewes. Journal Reproduction Fertility Supplement, n.49, p.393-407, 1995.

Kinder, J.E., Day, M.L. \& Kittok, R.J. Endocrine regulation of puberty in cows and ewes. Journal Reproduction Fertility Supplement, n.34, p.167-186, 1987.

Kinder, J.E., Roberson, M.S., Wolfe, M.W. \& Stumpf, T.T. Management factors affecting puberty in the heifer. In: Fields MJ, Sand RS (Ed.). Factors affecting calf crop. Boca Raton, FL: CRC Press, 1994. p.69-89.

Lobato, J.F.P. Sistemas intensivos de produção de carne bovina: I. Cria. In: Simpósio Sobre a Pecuária de Corte, 4, 1996, Piracicaba. Anais... Piracicaba: ESALQ, 1997. p.161-204.

Maciel M. N., Zieba D. A., Amstalden M., Keisler D. H., Neves J. P. \& Williams G. L. Chronic administration of recombinant ovine leptin in growing beef heifers: Effects on secretion of $\mathrm{LH}$, metabolic hormones, and 
timing of puberty. Journal Animal Science, v. 82, p. 2930-2936, 2004.

Marson, E.P.; Guimarães; J.D.; Guimaraes, S. E. F. ; Miranda Neto, T. Concentrações plasmáticas de progesterona em novilhas compostas Montana Tropical, durante as fases pré-puberal e puberal. Revista Brasileira de Reprodução Animal, v.25, p.134-136, 2001.

McDonald, L. E. Veterinary endocrinology and reproduction. 4th. Ed., Lea \& Febiger, Philadelphia, 1989.

Meireles, I. P. Influência do sombreado em parâmetros fisiológicos e produtivos de vacas mestiças (holandês x zebu). Itapetinga - BA: UESB, 2005. 63p. Dissertação (Mestrado em Zootecnia) - Universidade Estadual do Sudoeste da Bahia, 2005.

Mpntagnini, F. Sistemas agroflorestales: principios y aplicaciones en los tropicos, $2 \mathrm{a}$ ed. San Jose, CR. Organización para Estudios Tropicales. 622 p. 1992.

Nakada, K. et al. Changes in concentrations of plasma immunoreactive follicle-stimulating hormone. luteinizing hormone. estradiol-17 $\beta$. testosterone. progesterone. and inhibin in heifers from birth to puberty. Domestic Animal Endocrinology, v. 18, p. 57-69, 2000.

Nakada, K.; Morioyoshi, M. \& Nakao, T. Changes in peripheral levels of LH and FSH in prepubertal heifers after estradiol treatment. Journal of Reproduction and Development, v.47, n.6, p.341-349, 2001.

Nogueira, G. P. et al. Precocious fertility in Nelore heifers. Biology of Reproduction, Madison, v. 68 (S1), p. 382, 2003.

Nogueira, G.P. Puberdade e maturidade sexual de novilhas Bos indicus. Anais do $2^{\circ}$ Simpósio Internacional de Reprodução Animal Aplicada. Londrina-PR, 5 a 7 de Outubro de 2006.

Paciullo, D. S. C..; Carneiro, J. C.; Costa, F. J. N.; Tavela, R. C. \& Verneque, R. S.; Gomide, C .A. M. Massa de forragem, taxa de lotação e ganho de peso de novilhas mestiças em pastagem pura de Brachiaria decumbens e sistema silvipastoril. In: Reunión de la Asociación Latinoamericana de Producción Animal, 20.; 2007, Cusco. Anais... Cuzco, 2007.
Pires, M. F. A., Ferreira, A.M. \& Saturino, H.M. et al. Taxa de gestação em fêmeas da raça Holandesa confinadas em free stall, no verão e inverno. Arquivo Brasileiro de Medicina Veterinária. V. 54 n. 1. 2002.

Pires, M. F. A.; Novaes, L. P.; Mostaro, L. E. \& Souza, J. R. Temperatura retal e freqüência respiratória de vacas gir leiteiro, durante o verão. In: Reunião Anual da Sociedade Brasileira de Zootecnia, 42. 2005, Goiânia, GO. Anais... Goiânia: Sociedade Brasileira de Zootecnia, 2005.

Pires, M. F. A; Salla, L. E.; Paciullo, D. S. C.; Castro, C. R. T.; Aroeira, L. J. M.; Mostaro, L. E. Oliveira, M. C; Nascimento, F. J. Comportamento de novilhas mestiças Holandês x Zebu manejadas em pastagens de Brachiaria decumbens ou em sistema silvipastoril. In: Reunión de la Asociación Latinoamericana de Produción Animal, 20., 2007, Cuzco. Anais...Cuzco, 2007.

Rivier, C. \& Rivest, S. Effects of stresson the Activity of the Hypothalamic-PituitaryGonadal Axis: Peripheral and Central Mechanisms. Biology of Reproduction, v.45, p. 523-532, 1991.

Schillo, K. K. et al. Influence of season on sexual development in heifers: age at puberty as related to growth and serum concentrations of gonadotropins. prolactina. thyroxine and progesterone. Biology of Reproduction, Madison, v. 28, p. 329-341, 1983.

Terasawa, E. \& Fernadndez, D.F. Neurobiological mechanisms of the onset of puberty in primates. Endocrine Reviews, v.22, n.1. p.111-151. 2001.

Williams, G.L., Amstalden, M., Garcia, M.R., Stanko, R.L., Nizielski, S.E., Morrison, C.D., Keisler, D.H. Leptin and its role in the central regulation of reproduction in cattle. Domestic Animal Endocrinology, v.23, n.1, p.339-349, 2002.

Zieba D.A., Amstalden M., Williams G.L. Regulatory roles of leptin in reproduction and metabolism: A comparative review. Domestic Animal Endocrinology, v. 29, p. 166-185, 2005.

Recebido em Setembro 15, 2014

Aceito em Novembro 4, 2014 